\title{
Anti-Reflecting Surfaces Using Two-layer Motheye Structures for Spinel Ceramic Windows
}

\author{
Chaoran Tu and Curtis R. Menyuk \\ University of Maryland Baltimore County, Baltimore, MD 21250, USA \\ chaoran1@umbc.edu
}

\begin{abstract}
We design and optimize a two-layer pyramid motheye structures for long wavelength $\mathrm{MgAl}_{2} \mathrm{O}_{4}$ spinel ceramic windows. We show that a two-layer pyramid motheye structure can achieve average transmission of $98.96 \%$ from $0.5 \mu \mathrm{m}$ to $5 \mu \mathrm{m}$. $\odot 2018$ The Author(s)
\end{abstract}

OCIS codes: (050.6624) Subwavelength structures, (160.4670) Optical materials.

\section{Introduction}

Motheye structures are period biomimetic sub-wavelength structures (SWS) that are inspired by the eyes of nocturnal moths [1]. They can be used as anti-reflecting (AR) surfaces that can significantly reduce Fresnel reflections [2]. In previous work, Busse et al. [3] demonstrated transmission larger than $90 \%$ from $0.9 \mu \mathrm{m}$ to $1.6 \mu \mathrm{m}$ with transparent $\mathrm{MgAl}_{2} \mathrm{O}_{4}$ spinel ceramic windows. In this work, we propose a two-layer pyramid motheye structure to improve the transmission at visible light wavelengths. We computationally study the dependence of the motheye structure transmission on the dimensions of the parameters of the motheye structure using the finite-difference time-domain (FDTD) method [4], and we present a design for a dual motheye structure with an averaged transmission greater than $98.96 \%$ in the wavelength range of $0.5-5 \mu \mathrm{m}$.

\section{Numerical computation}

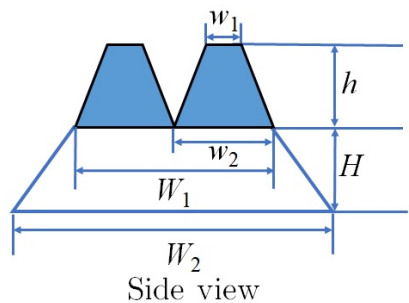

Side view

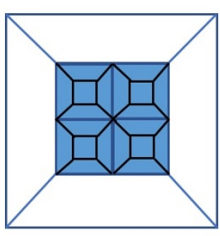

Top view

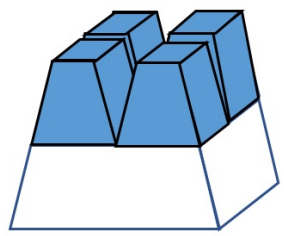

$3 \mathrm{D}$ view

Fig. 1. Illustration of the two-layer pyramid motheye structure.

In Fig. 1, we show a schematic illustration of the two-layer pyramid motheye structures that we will consider. This structure is characterized by six parameters, including the top width of the upper motheye layer $w_{1}$, the top width of the lower motheye layer $W_{1}$, the bottom width of the upper motheye layer $w_{2}$, the bottom width of the lower motheye layer $W_{2}$, the height of the upper motheye layer $h$, and the height of the bottom motheye layer $H$. The parameters of the lower motheye layer are equal to the experimental values in [3]: $W_{1}=0.3 \mu \mathrm{m}, W_{2}=0.6 \mu \mathrm{m}$ and $H=0.55 \mu \mathrm{m}$. The parameters of the upper motheye layer $w_{1}, w_{2}$ and $w_{3}$ vary over the range, $0.03-0.3 \mu \mathrm{m}, 0.03-0.3 \mu \mathrm{m}$, and $0.5-3.5 \mu \mathrm{m}$, respectively. The widths $w_{1}$ and $w_{2}$ are geometrically constrained by $W_{1}$, while the lower bound of $h$ is designed to maintain a high transmission at infrared wavelengths.

Fig. 2 shows the transmission spectra in which we vary $w_{1}, w_{2}$ and $h$ independently. We first vary $w_{1}$ from 0.03 $\mu \mathrm{m}$ to $0.3 \mu \mathrm{m}$ and fix $w_{2}=0.3 \mu \mathrm{m}$ and $h=3.5 \mu \mathrm{m}$. The transmission at visible light wavelengths increases when $w_{1}$ decreases. Next, we vary $w_{2}$ from $0.03 \mu \mathrm{m}$ to $0.3 \mu \mathrm{m}$ while fixing $w_{1}=0.03 \mu \mathrm{m}$ and $h=3.5 \mu \mathrm{m}$. The transmission spectra with $w_{2}=0.03 \mu \mathrm{m}$ is smaller than that in other cases by about $4 \%$ because most of the transmission incident light directly interacts with the lower layer. However, this structure can still achieve a broad bandwidth transmission spectrum. Finally we vary $h$ from $0.5 \mu \mathrm{m}$ to $3.5 \mu \mathrm{m}$ and fix $w_{1}=0.03 \mu \mathrm{m}$ and $w_{2}=0.3 \mu \mathrm{m}$. The transmission at the wavelengths from $0.5 \mu \mathrm{m}$ to $5 \mu \mathrm{m}$ increase as $h$ increases. By calculating the mean transmission over wavelengths 
between $0.5-5 \mu \mathrm{m}$, we find that the upper layer motheye structure with $w_{1}=0.03 \mu \mathrm{m}, w_{2}=0.3 \mu \mathrm{m}, h=3.5 \mu \mathrm{m}$ has an average transmission of 0.9896 .

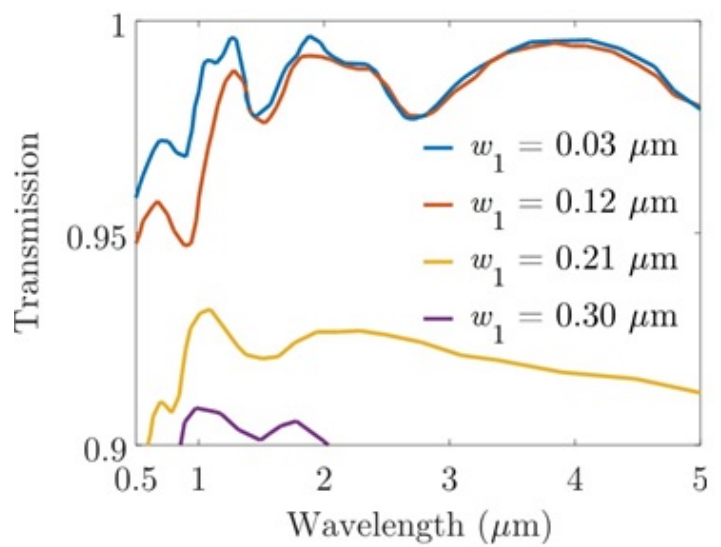

(a)

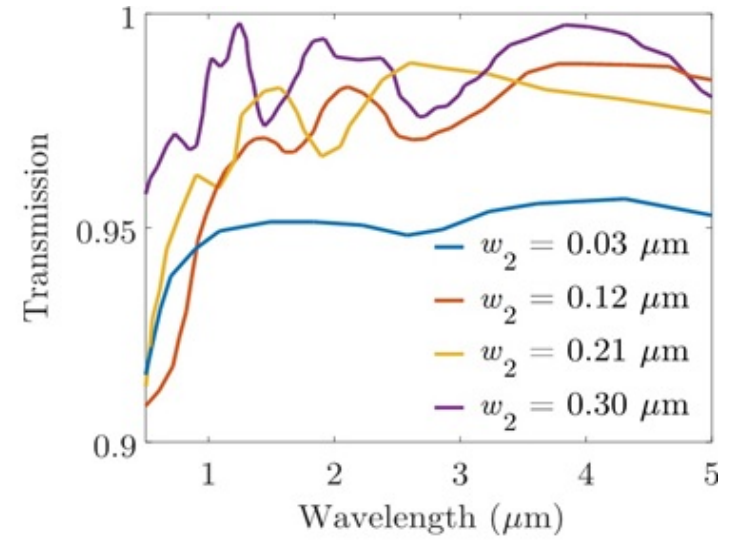

(b)

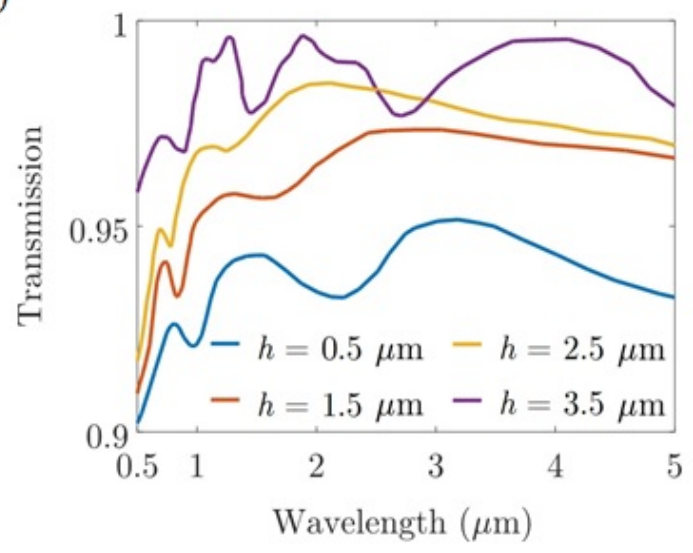

(c)

Fig. 2. The transmission spectra varying different parameters: (a) $h=0.28 \mu \mathrm{m}, w_{2}=0.3 \mu \mathrm{m} ; w_{1}$ varies from $0.03 \mu \mathrm{m}$ to $0.3 \mu \mathrm{m}$, (b) $h=0.28 \mu \mathrm{m}, w_{1}=0.03 \mu \mathrm{m}$; $w_{2}$ varies from $0.03 \mu \mathrm{m}$ to 0.3 $\mu \mathrm{m}$, (c) $x_{1}=0.03 \mu \mathrm{m}, w_{2}=0.28 \mu \mathrm{m} ; h$ varies from $0.5 \mu \mathrm{m}$ to $3.5 \mu \mathrm{m}$.

\section{Conclusion}

We studied two-layer pyramid motheye structures for transmission in the range $0.5-5 \mu \mathrm{m}$. We varied the dimensions of the motheye structures, and we showed that we could theoretically achieve almost $99 \%$ transmission over the entire range $0.5-5 \mu \mathrm{m}$. Our results demonstrated that high transmission in this entire wavelength range can be obtained by adding a second motheye layer.

\section{References}

1. C. G. Bernhard and William H. Miller, "A Corneal Nipple Pattern in Insect Compound Eyes," Acta Physiol. Scand. 56, 385-386 (1962).

2. D. G. Stavenga, S. Foletti, G. Palasantzas, and K. Arikawa,"Light on the moth-eye corneal nipple array of butterflies,", Proc. Biol. Sci. 273, 661-667 (2006).

3. Lynda E. Busse, Catalin M. Florea, Jesse A. Frantz, L. Brandon Shaw, Ishwar D. Aggarwal, Menelaos K. Poutous, Rajendra Joshi, and Jas S. Sanghera, "'Anti-reflective surface structures for spinel ceramics and fused silica windows, lenses and optical fibers," Opt. Mater. Express, 4, 2504-2515 (2014).

4. A. F. Oskooi, D. Roundy, M. Ibanescu, P. Bermel, J. D. Joannopoulos, and S. G. Johnson, "MEEP: A flexible free-software package for electromagnetic simulations by the FDTD method," Comp. Phys. Comm. 181, 687$702(2010)$. 